\title{
Free radical degradation of chitosan with potassium persulfate
}

\author{
Shih-Chang Hsu ${ }^{\mathrm{a}}$, Trong-Ming Don ${ }^{\mathrm{b}}$, Wen-Yen Chiu ${ }^{\mathrm{a}, *}$ \\ ${ }^{a}$ Department of Chemical Engineering, National Taiwan University, Taipei, Taiwan, ROC \\ ${ }^{\mathrm{b}}$ Department of Chemical Engineering, Mingchi Institute of Technology, Taipei Shein, Taiwan, ROC
}

Received 18 May 2001; received in revised form 23 July 2001; accepted 2 August 2001

\begin{abstract}
A thermal dissociation initiator, potassium persulfate (KPS), is added to the chitosan solution at $70{ }^{\circ} \mathrm{C}$; immediately, the solution viscosity and the molecular weight of chitosan decrease in a very short time. Size exclusion chromatography, nuclear magnetic resonance and electron spin resonance were used to study the degradation mechanism. A free radical degradation mechanism of chitosan by KPS is then proposed. When KPS is thermally dissociated into anionic radicals, they are attracted to the cationic amino group in the chitosan ring. Subsequently, the anionic radical attacks the C-4 carbon and transfers the radical to the C-4 carbon by subtracting the hydrogen from it. The presence of free radical at C-4 carbon eventually results in the breakage of the glycosidic C$\mathrm{O}-\mathrm{C}$ bond in the chitosan main chain. According to this mechanism, the concentrations of KPS, total free radicals and the degraded chitosan chain at different degradation times are all calculated by solving the rate equations. Finally, the calculated average molecular weights of the degraded chitosan chains at different reaction times agree with the experimental values. (C) 2001 Elsevier Science Ltd. All rights reserved.
\end{abstract}

Keywords: Chitosan; Potassium persulfate; Free radical; Degradation

\section{Introduction}

The potential use of polymeric nanoparticles as drug carriers in vivo has led to the research and development of many different drug delivery vehicles [1]. These drug carriers are required to be biocompatible, biodegradable, easy prepared and without any toxicity. One route to prepare them is by an emulsion polymerization process in which polymerization takes place almost exclusively in the micelles [2]. These active micelles where polymerization is occurring are then referred to as polymer particles. After reaction, their sizes are generally in the range of nanometers. Because in emulsion polymerization anionic surfactants are the most commonly used surfactants like potassium laurate or sodium lauryl sulfate, these nanoparticles are generally characterized by a negative surface charge. The negative charge helps to prevent the coalescence of nanoparticles. Yet, in vivo it promotes the adsorption of cationic substances present in the biological fluids, such as protein, sodium and calcium ions. As a result, this leads to the neutralization

\footnotetext{
* Corresponding author. Fax: + 886-2-23623259.

E-mail address: ycchiu@cc.ntu.edu.tw (W.-Y. Chiu).
}

and aggregation of nanoparticles and consequently, breakdown of the system. Therefore, positively charged nanoparticles may have the advantage in stability in the presence of biological cations [3]. In addition, they are probably favorable for some drugs due to the interaction with negatively charged biological membranes and site-specific targeting in vivo [4].

To synthesize positively charged nanoparticles, chitosan is chosen as a cationic surfactant in an emulsion polymerization. Chitosan is a high-molecular-weight polysaccharide composed of mainly of $\beta-(1,4)$ linked 2deoxy-2-amino-D-glucopyranose units and partially of $\beta$ - $(1,4)$ linked 2-deoxy-2-acetamido-D-glucopyranose. It is an environmentally friendly material and can be used in many areas, including wastewater treatment, food processing, pharmaceuticals, biomaterials and agriculture [5-7]. When dissolved in an acid solution, it becomes a cationic polymer due to the protonation of amino groups on the $\mathrm{C}-2$ position of pyranose ring. Therefore, it can be used as a cationic, polymeric surfactant, which offers both electrostatic and steric stabilization of dispersion particles. Chern et al. [8,9] have studied the colloidal stability of positively charged poly(methyl methacrylate) (PMMA) latex particles 
using chitosan as a cationic surfactant. They suspected that chitosan has been incorporated into PMMA molecules; yet they did not take any further investigation of the reaction mechanism. Yang et al. [10] also have synthesized positively charged poly(butyl cyanoacrylate) nanoparticles stabilized with chitosan.

In this article, potassium persulfate (KPS) is used as an initiator for monomer polymerization with the help of chitosan surfactant. The monomer solution initially is very viscous because of the presence of high-molecularweight chitosan. Yet, when KPS is added and thermally dissociated into free radicals at $70{ }^{\circ} \mathrm{C}$, the solution viscosity decreases sharply in a very short time. Before examining structures and properties of the final polymer particles, it is interesting and important to investigate the mechanism behind the decrease of solution viscosity, because it would have effects on the polymerization rate, structures and properties of the final polymer particles. A reasonable assumption is that the decrease in solution viscosity is due to the degradation of chitosan molecules. In addition, it is well known that the functional properties of chitosan materials have been found to depend on their molecular weight. Therefore, the efficacy of chitosan to coagulate pollutants, promote antimicrobial activity, control viscosity, accelerate burn healing, lower blood cholesterol levels and improve crop yields depends on this specific characteristic. Especially, chitosan oligomers or chito-oligosaccharides are of special interest in agriculture and medicine $[11,12]$. They have high activities at a very low concentration. Therefore, the development of the methods for preparing these oligosaccharides is attracting growing interest. There are several methods to degrade chitosan molecules including enzymatic hydrolysis, acid hydrolysis, highenergy irradiation, thermal degradation and ultrasonic treatment [13-16]. Therefore, it is also beneficial to study the degradation behavior of chitosan by KPS from this aspect. In this article, we thus present the detailed studies on the degradation behavior of chitosan by KPS, whereas the structures and properties of polymer particles will be described in another article.

\section{Experimental}

\subsection{Materials}

Chitosan (Tokyo Chemicals Inc., Tokyo) was purified before use. It was first dissolved into a $2 \%$ acetic acid solution, and then precipitated out by adding a concentrated $\mathrm{NaOH}$ solution. The precipitate was washed several times with a great amount of deionized water until the $\mathrm{pH}$ had reached 7 . The degree of deacetylation of chitosan was found to be $86 \%$ by a colloid titration method [17]. In this method, a specific amount of chitosan-acetic acid solution was titrated with $0.0025 \mathrm{~N}$
PVSK (potassium polyvinylsulfate, $\left(\mathrm{C}_{2} \mathrm{H}_{3} \mathrm{O}_{4} \mathrm{SK}\right)_{n}$, $n=1500$ or above), where $0.1 \%$ toluidine blue was used as an indicator. KPS was a reagent grade from Wako Pure Chemical Industries, Osaka. All the other chemicals were analytical-grade and used as received without further purification.

\subsection{Degradation of chitosan by KPS}

In a round-bottomed reaction flask purged with nitrogen, 11 of $2 \%(\mathrm{w} / \mathrm{w})$ acetic acid solution and a specific amount of chitosan powder were added serially. After thoroughly stirring, chitosan was completely dissolved into the acetic acid solution. It was then heated to $70{ }^{\circ} \mathrm{C}$ in an isothermal water bath and stirred at a speed of $300 \mathrm{rpm}$. Subsequently, $1.08 \mathrm{~g}$ of KPS was added to the solution and immediately started the degradation of chitosan. A small amount of the degraded chitosan solution was drawn out from the reaction flask for the measurement of molecular weight and viscosity.

\subsection{Molecular weight of chitosan}

The molecular weight of chitosan was determined by a viscometric method using an Ostwald viscometer at $25{ }^{\circ} \mathrm{C}$. Several different concentrations were prepared by the dilution of the degraded chitosan solution with a $2 \% \mathrm{CH}_{3} \mathrm{COOH}-0.2 \mathrm{M} \mathrm{CH}_{3} \mathrm{COONa}$ solution. The specific viscosity $\left(\eta_{\mathrm{sp}}\right)$ at different concentrations was then measured and the intrinsic viscosity $([\eta])$ was obtained by extrapolating the linear plot of $\eta_{\mathrm{sp}} / c$ vs. $c$ to the zero concentration. The viscosity average molecular weight $\left(\overline{M_{v}}\right)$ was then calculated using the Mark-Houwink equation, $[\eta]=k{\overline{M_{\mathrm{V}}}}^{\alpha}$, in which the constants $k$ and $\alpha$ depend on the degree of deacetylation [18,19]. Molecular weight was also determined by a size-exclusion high-performance liquid chromatography (SE-HPLC) following the method of Yomota et al. [20]. The mobile phase consisted of $0.2 \mathrm{M} \quad \mathrm{CH}_{3} \mathrm{COOH}-0.1 \mathrm{M}$ $\mathrm{CH}_{3} \mathrm{COONa}$, and $0.008 \mathrm{M} \mathrm{NaN}_{3}$. Sample concentration of $0.1 \%(\mathrm{w} / \mathrm{v})$ was loaded and eluted with a flow rate of $0.6 \mathrm{ml} / \mathrm{min}$. Two serial columns packed with TSK gel G4000 $\mathrm{PW}_{\mathrm{XL}}$ and G5000 $\mathrm{PW}_{\mathrm{XL}}$ (Tosoh, Japan) were used. The elution peak was detected by a differential refractometer (Gilson, model M132, USA).

\subsection{Structure identification}

The nuclear magnetic resonance (NMR) spectra were obtained with a $300 \mathrm{MHz}$ solid-state NMR spectrometer (Bruker, MSL-300). Samples were ground to a fine powder and scanned with a CP/MAS technique at a frequency of $75.47 \mathrm{~Hz}$. An electron spin resonance (ESR) spectrometer from Bruker (model ESP 300 Xband) was used to detect the free radicals produced during the degradation of chitosan. $N$-phenyl-t-butylnitrone 
(PBN) was used as the spin-trapping reagent and 2,2diphenylpicrylhydrazyl was used as the internal standard.

\section{Results and discussion}

After adding KPS into the chitosan solution at $70{ }^{\circ} \mathrm{C}$, the solution viscosity decreased rapidly in a short time. The intrinsic viscosity ([ $\eta]$ ) of degraded solution and the corresponding viscosity average molecular weight $\left(\overline{M_{v}}\right)$ of chitosan decreased quickly at first 5 min and already reached a plateau value at only $10 \mathrm{~min}$, as listed in Table 1. This indicates that KPS is a very effective reagent in degrading the chitosan. KPS, when heated, would decompose into two anionic free radicals, $\mathrm{S}_{2} \mathrm{O}_{8}^{2-} \rightarrow 2 \cdot \mathrm{SO}_{4}^{-}$. Since salts like sodium sulfate, potassium carbonate and sodium chloride, which do not degrade chitosan, it is thought that the free radicals are responsible for the degradation of chitosan. Furthermore, several other free radical initiators were used to test their efficacy under the same reaction conditions, including 4,4'-azobis(4-cyanovaleric acid) (ABCVA), benzoyl peroxide (BPO) and cerium ammonium nitrate $(\mathrm{CAN})$. ABCVA and BPO are both thermally dissociated initiators like KPS, yet ABCVA is an organic acid and BPO is hardly soluble in water. Though CAN is water-soluble, it is a redox initiator rather than a thermally dissociated initiator, where it oxides the glucopyranose ring to produce free radical directly on the ring. Therefore, it is generally used in graft polymerization of monomers onto polysaccharides. With ABCVA and BPO, the decrease in solution viscosity was only observable after $90 \mathrm{~min}$. Yet, with CAN initiator, there is no change in solution viscosity even after $5 \mathrm{~h}$. Therefore, the degradation of chitosan can be initiated most effectively by the anionic free radicals present in the aqueous solution. Fig. 1 shows the SE-HPLC chromatograms of chitosan during the KPS degradation. In

Table 1

Intrinsic viscosity and the corresponding average molecular weight of chitosan at different degradation times ${ }^{\mathrm{a}}$

\begin{tabular}{lcrrr}
\hline $\begin{array}{l}\text { KPS } \\
\text { degradation } \\
(\mathrm{min})\end{array}$ & $\begin{array}{l}\text { Intrinsic } \\
\text { viscosity }[\eta] \\
(\mathrm{ml} / \mathrm{g})\end{array}$ & $\begin{array}{l}\bar{M}_{\mathrm{v}} \mathrm{b} \\
(\mathrm{g} / \mathrm{mol})\end{array}$ & \multicolumn{1}{l}{$\begin{array}{l}\overline{M_{\mathrm{n}}} \mathrm{c} \\
(\mathrm{g} / \mathrm{mol})\end{array}$} & $\overline{M_{\mathrm{v}}} / \overline{M_{\mathrm{n}}}$ \\
\hline 0 & 1150.6 & 615,900 & 205,300 & 3.00 \\
5 & 247.7 & 101,100 & 17,800 & 5.68 \\
10 & 77.4 & 25,700 & 9900 & 2.59 \\
20 & 67.7 & 22,000 & 6200 & 3.54 \\
30 & 64.8 & 20,900 & 5300 & 3.93 \\
45 & 57.0 & 17,900 & 5000 & 3.59 \\
60 & 55.6 & 17,400 & 5000 & 3.51 \\
\hline
\end{tabular}

a $1.08 \mathrm{~g}$ of KPS was added into 11 of $2 \%(\mathrm{w} / \mathrm{w})$ acetic acid solution with 15 -g chitosan. The temperature was $70{ }^{\circ} \mathrm{C}$.

b From the Mark-Houwink equation, $[\eta]=k{\overline{M_{\mathrm{v}}}}^{\alpha}, k=1.38 \times 10^{-2}$, $\alpha=0.85$ [Ref. 16].

${ }^{c}$ From the calculation of Eq. (13).
SE-HPLC chromatogram, those with higher molecular weights would have less retention times $\left(t_{\mathrm{R}}\right)$. Therefore, the results in Fig. 1 indicate that the molecular weight of chitosan decreased with degradation time. It is generally assumed in the literature [2] that there is a linear relationship between the logarithm of number average molecular weight and the retention time: $\log \overline{M_{\mathrm{n}}}=-a$ $\left(t_{\mathrm{R}}\right)+b$. The results will be used further to verify the degradation mechanism proposed in the following section.

A solid-state ${ }^{13} \mathrm{C}$-NMR spectrometer was used to observe the structure changes of chitosan after degradation. Table 2 lists all the resonance peaks of carbons in the chitosan, Scheme 1, before and after KPS degradation. There was almost no change in the spectrum after degradation except the absorption peak of C-1 carbon shifted to $98 \mathrm{ppm}$. This strongly suggested that under degradation the electron density around the C-1 carbon had changed, and, therefore, breakage and

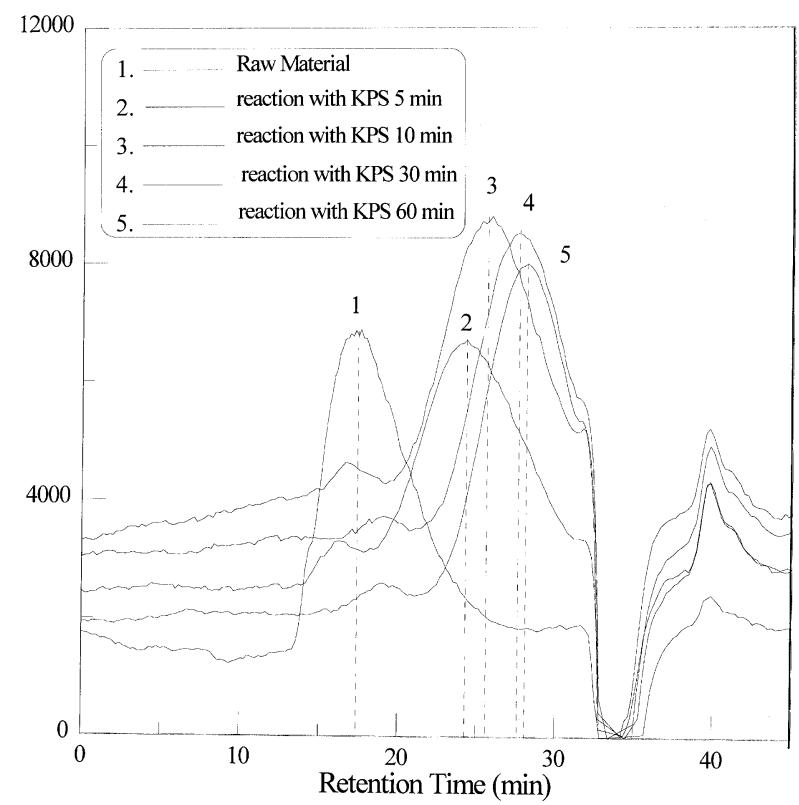

Fig. 1. Size exclusion chromatograms of chitosan at different degradation times. $15 \mathrm{~g}$ of chitosan in a $2 \%(\mathrm{w} / \mathrm{w})$ acetic acid solution was degraded by $1.08 \mathrm{~g}$ of KPS at $70{ }^{\circ} \mathrm{C}$.

Table 2

${ }^{13} \mathrm{C}-\mathrm{NMR}$ chemical shift of various carbons in the chitosan before and after degradation

\begin{tabular}{lcc}
\hline Chitosan & \multicolumn{2}{c}{ Chemical shift $(\mathrm{ppm})$} \\
\cline { 2 - 3 } & Before degradation & After degradation $^{\mathrm{a}}$ \\
\hline C1 & 102 & 98 \\
C2, C6 & $53-59$ & $53-59$ \\
C3, C4 & $71-81$ & $71-81$ \\
C7 & 20 & 21 \\
C8 & 173 & 172
\end{tabular}

a Fifteen grams of chitosan was degraded by $1.08 \mathrm{~g}$ of KPS for $1 \mathrm{~h}$ at $70{ }^{\circ} \mathrm{C}$ in a $2 \%(\mathrm{w} / \mathrm{w})$ acetic acid solution. 
formation of chemical bonds most probably occurred at or close to the $\mathrm{C}-1$ carbon. The previous results have shown that the degradation leads to the chain scission, and the chitosan main chain is composed of pyranose rings linked by $\beta-(1,4)$ glycosidic bond. It is then reasonable to believe that the KPS free radical would attack the $\mathrm{C}-1$ or $\mathrm{C}-4$ carbon and then break the adjacent $\mathrm{C}-\mathrm{O}-\mathrm{C}$ glycosidic bond in the main chain. Though the electron density around $\mathrm{C}-4$ carbon should also be changed, but it was not clearly observed in the NMR spectrum because the peak was broad and overlapped with some other carbons.

Electron spin resonance (ESR) has been mainly used for detection of radical species involved in polymerization, degradation and oxidation. The presence of unpaired electrons is prerequisite to obtaining an ESR spectrum. Yet, when radical species are at too low a concentration or have too short a lifetime for ESR detection, they may be converted to an ESR detectable product called a spin-adduct by reaction with a spintrapping agent. For this reason, a spin-trapping agent, phenyl- $N$ - $t$-butylnitrone $(\mathrm{PBN})$, was used to react with any radicals present in the reaction system. The reaction is shown in Scheme 2.

After thermal treatment at $70{ }^{\circ} \mathrm{C}$, the ESR spectra of $\mathrm{PBN}, \mathrm{PBN}+\mathrm{KPS}$, and $\mathrm{PBN}+\mathrm{KPS}+$ chitosan are shown in Fig. 2(a)-(c), respectively. Compared with the spectrum of $\mathrm{PBN}$, some additional but very small absorption signals were observed in the PBN + KPS system, which was believed due to the spin-adduct formed by PBN with the free radicals dissociated from KPS. The ESR spectrum shown in Fig. 2(c) indicated that as chitosan was degraded by KPS free radicals, some new radicals were regenerated on the chitosan chains.

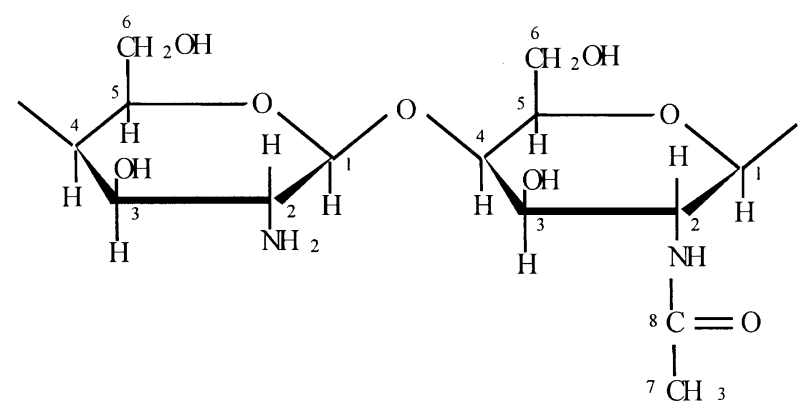

Scheme 1. Various carbons in the chitosan.
From the above results, a free radical degradation mechanism of chitosan by KPS is proposed in the following. When KPS was thermally dissociated into anionic radicals, they were very likely to be attracted to the cationic amino groups $\left(\mathrm{NH}_{3}^{+}\right)$at the $\mathrm{C}-2$ carbon of the chitosan ring because of the electrostatic attraction. Subsequently, the anionic radical would attack the C-4 carbon and transferred the radical to the $\mathrm{C}-4$ carbon by subtracting the hydrogen from it. The presence of a free radical at the $\mathrm{C}-4$ carbon would have weakened and homolytically broken the next $\mathrm{C}-\mathrm{O}$ bond at the $\mathrm{C}-1$ carbon and transfer the free radical to the $\mathrm{C}-1$ carbon. Consequently, the chitosan chain was degraded into two shorter chains. One had a free radical on the C-1 carbon at the end, and the other had a carbonyl group at the C-4 carbon in the terminal ring. Therefore, the NMR absorption peak of the C-1 carbon was shifted form 102 to 98 ppm. Though, it was possible that the KPS free radical would attack the C-1 carbon and turned the two shorter chains the other way around. If this were true, then the terminal C-1 carbon would become a carbonyl group and would have a higher chemical shift value. Yet, this was not supported by the NMR results. Nevertheless, the chitosan chain was getting shorter and shorter through the scission of $\mathrm{C}-\mathrm{O}-\mathrm{C}$ bonds in the main chain. The reaction scheme is shown in Scheme 3.

The degradation of chitosan by KPS was very fast and almost complete after $1 \mathrm{~h}$, as revealed in the viscosity changes. Yet, the dissociation rate constant of KPS at $70{ }^{\circ} \mathrm{C}$ is only $9.23 \times 10^{-6} 1 / \mathrm{s}$, which means the half-life time of KPS is about $20.9 \mathrm{~h}$ [2]. That means after $1 \mathrm{~h}$ of degradation a lot of KPS would still remain in the solution. If a vinyl monomer was added for polymerization to the solution after $1 \mathrm{~h}$ degradation, it still could be initiated by the new free radicals dissociated from the remaining KPS. Yet, there was no polymerization, when methyl methacrylate was added to the degraded chitosan solution. This leads to a suspicion that the degraded chitosan chains could inhibit the free radicals or deactivate the persulfate ions. These reactions are also shown in the reaction scheme, reactions (4) and (5). It was thought that the degraded chain ends with carbonyl group at C-4 carbon, CS, could react with any free radicals present in the aqueous solution and changed them to the products without any activity. It is also believed that after degradation, the persulfate anions were much more easily attracted to the<smiles>[R]C[C@H]1C=CC=C(C([R])([2H])N([O])C(C)(C)C)C1</smiles>

Scheme 2. The reaction of a spin-trapping agent, phenyl- $N$-t-butylnitrone $(\mathrm{PBN})$, with a radical. 
(1) Thermal dissociation of KPS

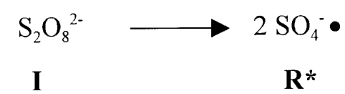

(2) Persulfate ion was attracted to the cationic amino group and free radical was transferred to the $\mathrm{C}-4$ carbon<smiles>[R20]S(=O)(=O)CC</smiles>

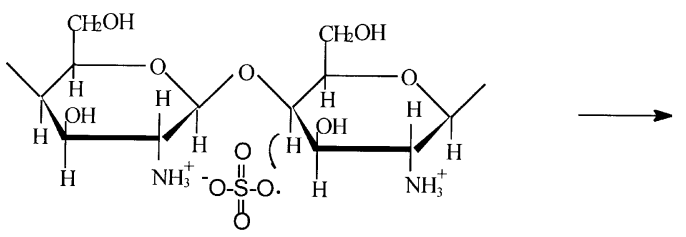

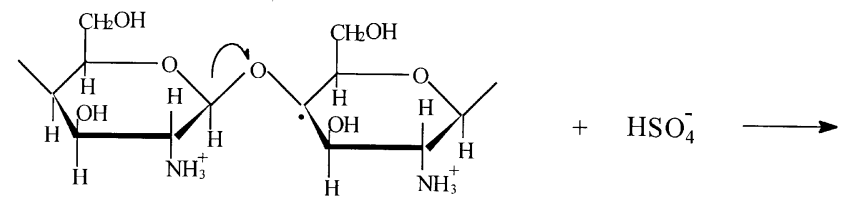

(3) Chain scission at the $\mathrm{C}-\mathrm{O}-\mathrm{C}$ bond

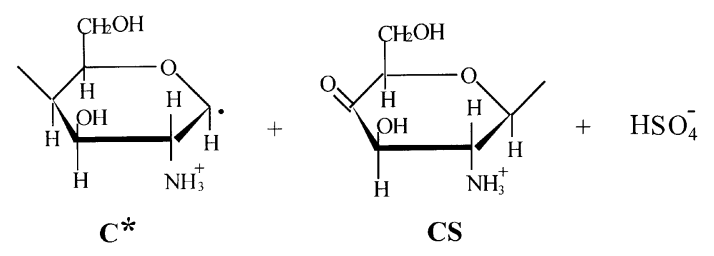

(4) Inhibition of free radical by degraded chitosan chain with carbonyl group at the end

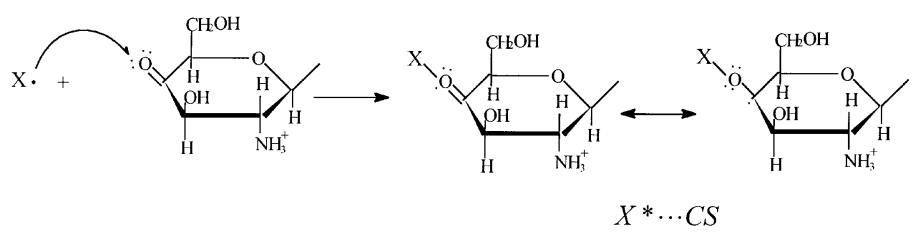

(5) Deactivation of persulfate ions

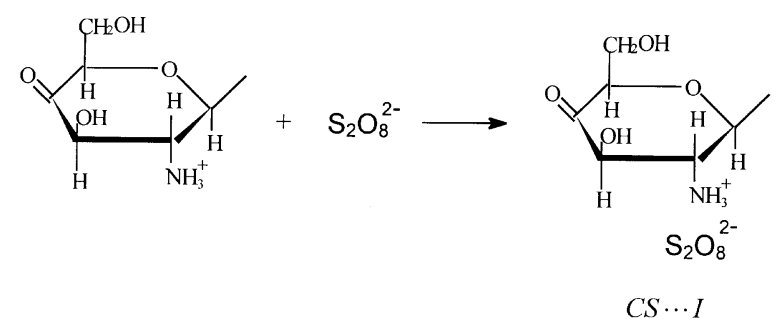

Scheme 3. Proposed degradation mechanism of chitosan by KPS free radical. 

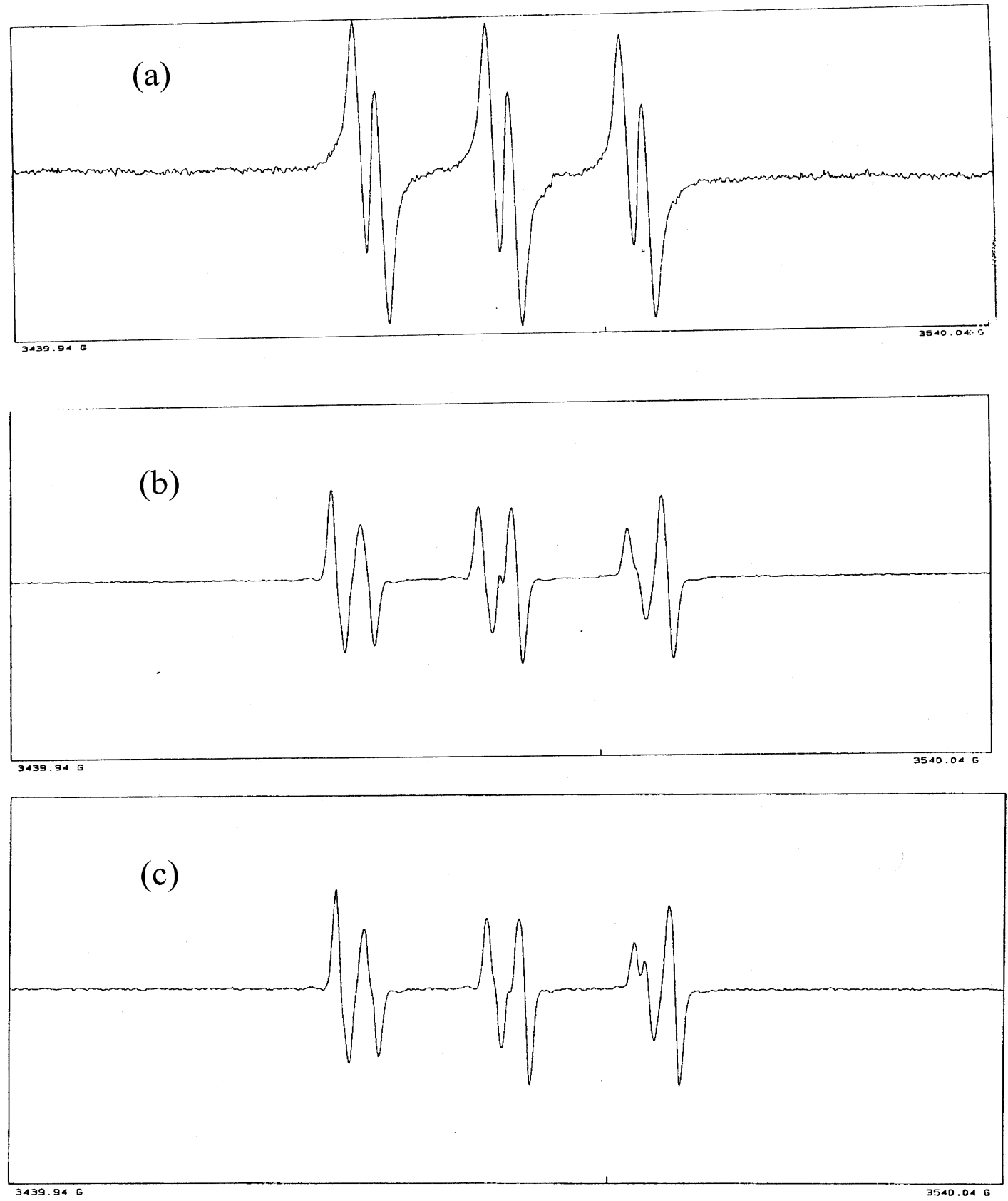

Fig. 2. ESR spectra of PBN (a), PBN + KPS (b), and PBN + KPS + chitosan (c). $15 \mathrm{~g}$ of chitosan in a $2 \%$ (w/w) acetic acid solution was degraded by $1.08 \mathrm{~g}$ of KPS at $70^{\circ} \mathrm{C}$.

cationic amino groups in the chitosan, since the solution viscosity was reduced greatly. Therefore, the ionic force immobilized the persulfate ions onto the degraded chitosan chains. As a result, a pronounced "cage effect" was observed that the dissociation of persulfate ion into two radicals were held within the chitosan cage. The trapped radicals then underwent recombination before initiating any monomers. As a result, the feeding monomer did not undergo any polymerization. On the contrary, if fresh KPS was added again following the addition of monomer, polymerization reaction was observed to take place. This is because the newly added KPS was freely
Table 3

Parameters needed to solve the rate equations ${ }^{\mathrm{a}}$

\begin{tabular}{lc}
\hline KPS concentration $\left[I_{\mathrm{o}}\left(\mathrm{mol} / \mathrm{l}-\mathrm{H}_{2} \mathrm{O}\right)\right.$ & 0.004 \\
Molecular weight of chitosan repeating & 183 \\
unit including glycosidic oxygen, $(\mathrm{g} / \mathrm{mol})$ & \\
$k_{\mathrm{d}}(1 / \mathrm{s})$ & $9.23 \times 10^{-6}$ \\
$f$ & 0.9 \\
$\overline{k_{\mathrm{t}}}(1 / \mathrm{mol} / \mathrm{s})$ & $2.69 \times 10^{7}$ \\
$\overline{k_{\mathrm{tr}}}(1 / \mathrm{mol} / \mathrm{s})$ & 50 \\
$k_{\mathrm{de}}(1 / \mathrm{mol} / \mathrm{s})$ & 1 \\
$\overline{k_{\mathrm{dC}}}(1 / \mathrm{mol} / \mathrm{s})$ & 650 \\
\hline
\end{tabular}

a $\overline{k_{\mathrm{tr}}}, k_{\mathrm{de}}$ and $\overline{k_{\mathrm{dC}}}$ are adjustable parameters in order to agree with the experimental values. 
to initiate monomer for polymerization, before they were trapped inside the degraded chitosan chains.

According to the above mechanism, the kinetic reactions are listed below.

(1) Initiation and deactivation of KPS

$I \stackrel{k_{\mathrm{d}}}{\rightarrow} 2 R^{*}$

$I+\mathrm{CS} \stackrel{k_{\mathrm{de}}}{\rightarrow} \mathrm{CS} \cdots I$

$I \quad$ : KPS initiator

$R^{*} \quad: \quad$ free radical produced from the KPS dissociation

CS : degraded chitosan chain fragment with a terminal carbonyl group

CS $\cdots I$ : deactivated KPS

$k_{\mathrm{d}} \quad: \quad$ the dissociation rate constant of KPS (1/s)

$k_{\mathrm{de}} \quad: \quad$ the deactivation rate constant of KPS $(\mathrm{l} / \mathrm{mol} / \mathrm{s})$

(2) Degradation of chitosan chains

$R^{*}+\mathrm{C}-\mathrm{O}-\mathrm{C} \stackrel{k_{d \mathrm{R}}}{\rightarrow} \mathrm{CS}+C^{*}$

$\mathrm{C}^{*}+\mathrm{C}-\mathrm{O}-\mathrm{C} \stackrel{k_{d c}}{\rightarrow} \mathrm{CS}+C^{*}$
$\mathrm{C}-\mathrm{O}-\mathrm{C}$ : the $\beta-(1,4)$ glycosidic bond in the chitosan chain

$C^{*} \quad$ : degraded chitosan fragment with free radical at the end

$k_{\mathrm{dR}}, k_{\mathrm{dC}}:$ the degradation rate constants $(1 / \mathrm{mols})$

(3) The inhibition of free radicals by the degraded chitosan chains

$R^{*}+\mathrm{CS}^{k_{t r \mathrm{R}}} \rightarrow R^{*} \ldots \mathrm{CS}$

$C^{*}+\mathrm{CS}^{k_{t r C}} \rightarrow C^{*} \cdots \mathrm{CS}$

$R^{*} \ldots \mathrm{CS}$ and $C^{*} \ldots \mathrm{CS} \quad: \quad$ stable free radicals

$k_{\mathrm{trR}}, k_{\mathrm{trC}}$ the inhibition rate constants of free radicals $(1 / \mathrm{mols})$

The total free radicals $\left(R_{\mathrm{t}}^{*}\right)$ in the water consisted of anionic radicals from KPS and degraded chitosan chain radicals, i.e. $R_{\mathrm{t}}^{*}=R^{*}+C^{*}$.

(4) Termination of free radicals

Collisions between free radicals and/or oligomeric free radicals could result in the termination of themselves.

$R_{\mathrm{t}}^{*}+R_{\mathrm{t}}^{*} \stackrel{\overline{k_{\mathrm{t}}}}{\rightarrow} R_{\mathrm{t}}-R_{\mathrm{t}}$

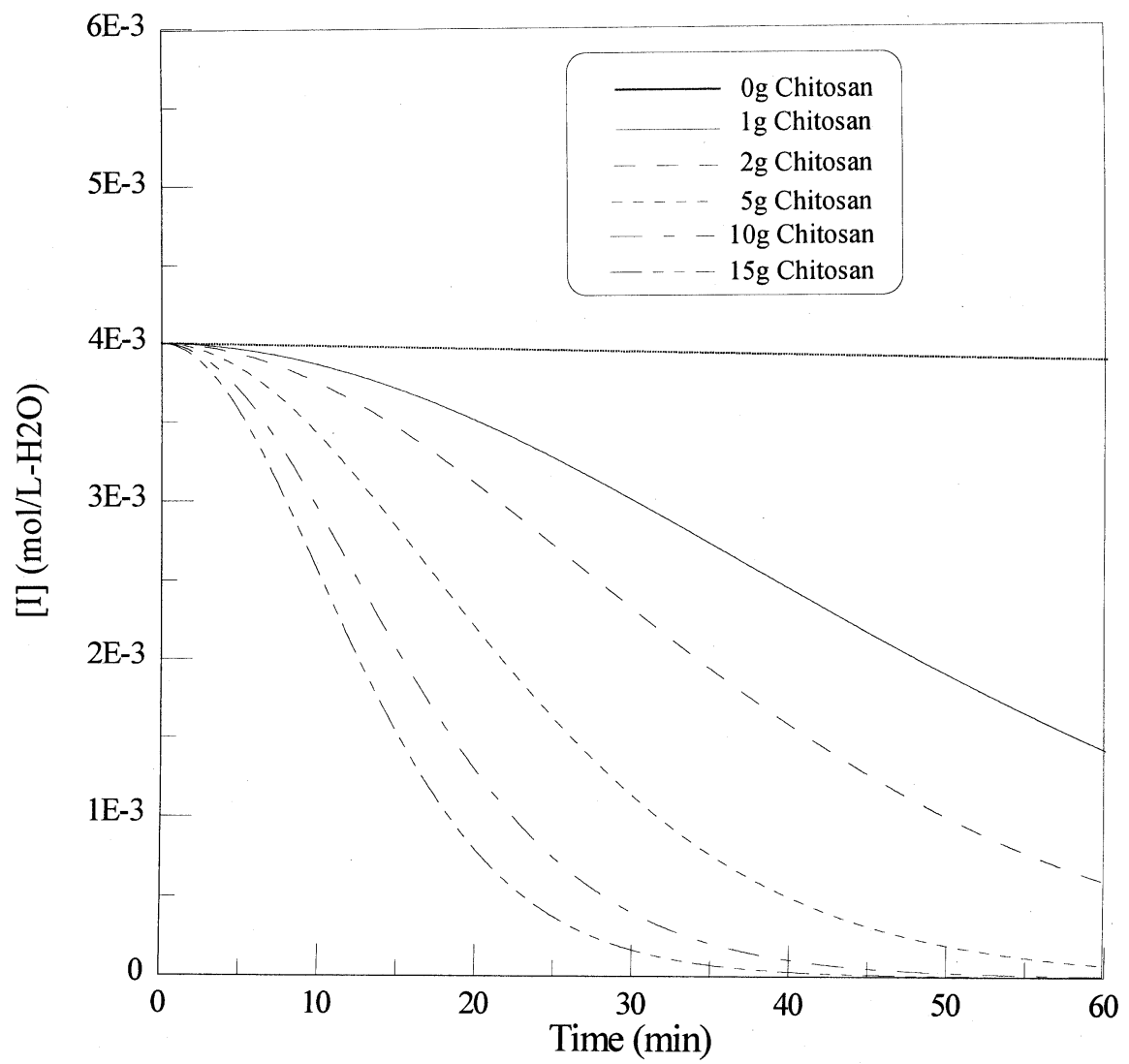

Fig. 3. Theoretical concentrations of initiator with degradation time at different amounts of chitosan added. KPS was $1.08 \mathrm{~g}$ and the temperature was $70{ }^{\circ} \mathrm{C}$. 
$\overline{k_{\mathrm{t}}} \quad$ : the average termination rate constant of free radicals $(1 / \mathrm{mol} / \mathrm{s})$

From the above kinetic reactions, the rate equations can be expressed as follows. First, the disappearance rate of the initiator is obtained by a combination of Eqs. (1) and (2).

$\frac{-\mathrm{d}[I]}{\mathrm{d} t}=k_{\mathrm{de}}[\mathrm{CS}][I]+k_{\mathrm{d}}[I]$

From Eqs. (3) and (4), the production rate of degraded chitosan chain CS is:

$\frac{\mathrm{d}[\mathrm{CS}]}{\mathrm{d} t}=\overline{k_{\mathrm{dC}}}[\mathrm{C}-\mathrm{O}-\mathrm{C}]\left[R_{\mathrm{t}}^{*}\right]$

$[\mathrm{C}-\mathrm{O}-\mathrm{C}]=[\mathrm{C}-\mathrm{O}-\mathrm{C}]_{\mathrm{o}}-[\mathrm{CS}]$

$[\mathrm{C}-\mathrm{O}-\mathrm{C}]_{\mathrm{O}}$ : the initial concentration of the glycosidic $\mathrm{C}-\mathrm{O}-\mathrm{C}$ unit $(\mathrm{mol} / \mathrm{l})$.

[C-O-C] : the concentration of the $\mathrm{C}-\mathrm{O}-\mathrm{C}$ unit (mol/l)

$\overline{k_{\mathrm{dC}}} \quad:$ the average degradation rate constant $(\mathrm{l} / \mathrm{mol} / \mathrm{s})$
The rate of change of total free radicals can be expressed as:

$\frac{\mathrm{d}\left[R_{\mathrm{t}}^{*}\right]}{\mathrm{d} t}=2 k_{\mathrm{d}} f[I]-\overline{k_{\mathrm{t}}}\left[R_{\mathrm{t}}^{*}\right]^{2}-\overline{k_{\mathrm{tr}}}\left[R_{\mathrm{t}}^{*}\right][\mathrm{CS}]$

$\frac{f}{k_{\mathrm{tr}}} \quad: \quad$ the efficiency of free radicals

$\overline{k_{\mathrm{tr}}} \quad: \quad$ the average inhibition rate constant of free radicals $(1 / \mathrm{mols})$

If a quasi-steady state approximation was assumed, Eq. (10) is then equal to zero.

$2 k_{\mathrm{d}} f[I]-\overline{k_{\mathrm{t}}}\left[R_{\mathrm{t}}^{*}\right]^{2}-\overline{k_{\mathrm{tr}}}\left[R_{\mathrm{t}}^{*}\right][\mathrm{CS}]=0$

The concentration of total free radicals thus can be obtained by solving the above equation.

$\left[R_{\mathrm{t}}^{*}\right]=\frac{-\overline{k_{\mathrm{tr}}}[\mathrm{CS}]+\sqrt{\left(\overline{k_{\mathrm{tr}}}[\mathrm{CS}]\right)^{2}+8 \overline{k_{\mathrm{t}}} k_{\mathrm{d}} f[I]}}{2 \overline{k_{\mathrm{t}}}}$

To solve the simultaneous equations [Eqs. (8), (9) and (12)], the Runge-Kutta method was used. All the

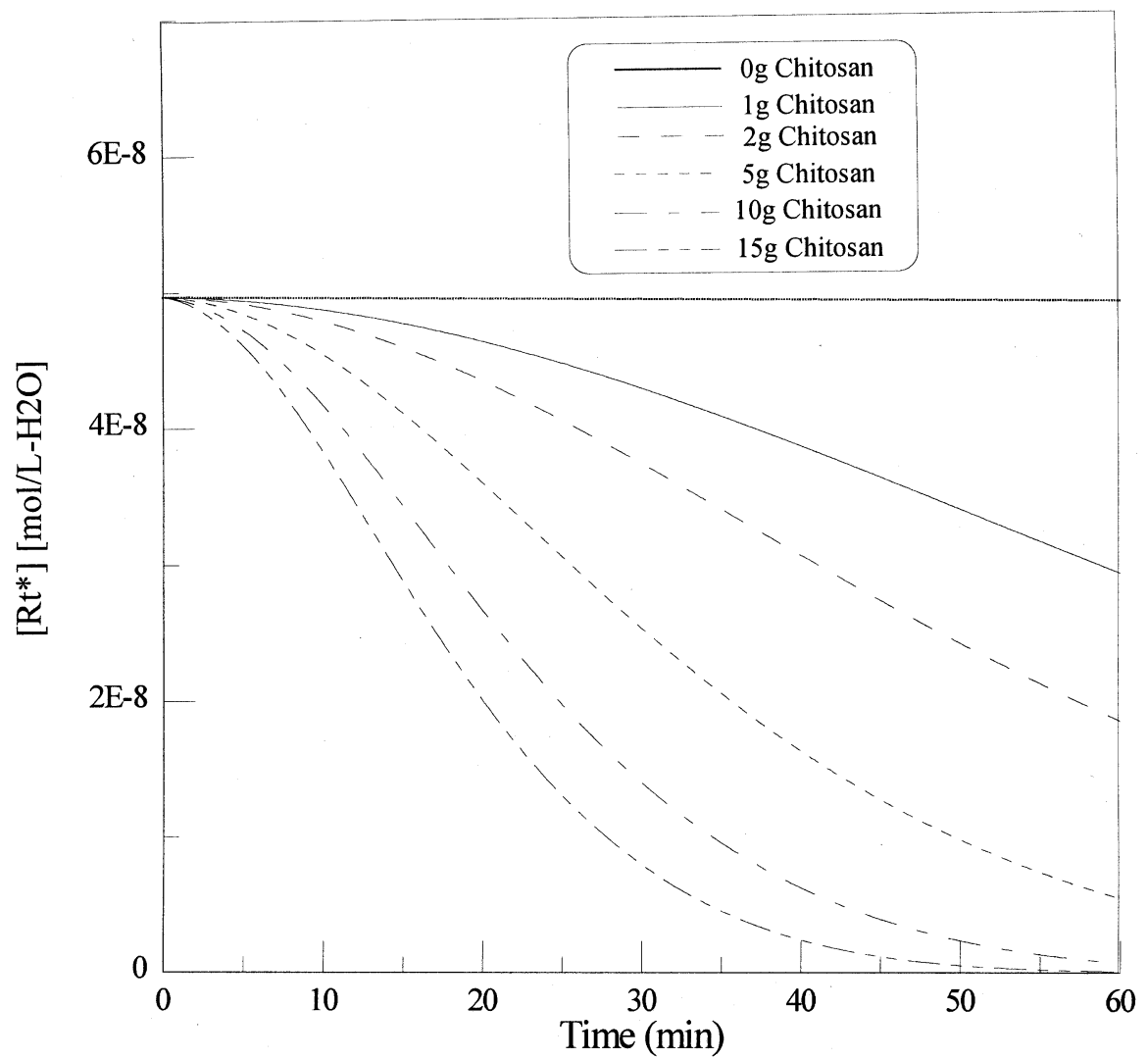

Fig. 4. Theoretical concentrations of total free radicals with degradation time at different amounts of chitosan added. KPS was $1.08 \mathrm{~g}$ and the temperature was $70{ }^{\circ} \mathrm{C}$. 
parameters needed to solve the equations were taken from the literature [2,21-23] and were listed in Table 3. By using these parameters, the theoretical concentrations of different species with reaction time were calculated at different amount of chitosan addition. Obviously, as shown in Figs. 3-5, the concentrations of initiator $[I]$ and total free radicals $\left[R_{\mathrm{t}}^{*}\right]$ in the aqueous system decreased with time, where an increase was observed for the degraded chitosan fragment [CS]. In addition, increasing the amount of initial chitosan accelerated the decreasing rate of free radical concentration. With $15 \mathrm{~g}$ of chitosan, there was no more free radical available in the system after $1 \mathrm{~h}$ of degradation. Consequently, if a monomer was added afterwards, no polymerization could be observed.

From the above mechanism, it is known that the number of chitosan fragment with carbonyl group at the end, $N_{\mathrm{CS}}$, which was obtained from the curves in Fig. 5, is equivalent to the times of chain scission. That is:

$\frac{W}{\overline{M_{\mathrm{n}}}}-\frac{W}{\overline{M_{\mathrm{no}}}}=N_{\mathrm{CS}}$
In the equation, $W$ is the weight of chitosan; $\overline{M_{\text {no }}}$ and $\overline{M_{\mathrm{n}}}$ are the number average molecular weights of original and degraded chitosan, respectively. Since the measured viscosity average molecular weight of original chitosan $\left(\overline{M_{\mathrm{v}}}\right)$ is 615,900 , the $\overline{M_{\text {no }}}$ value of chitosan would be 154,000 if the polydispersity index (PDI), $\overline{M_{\mathrm{v}}}$ $\overline{M_{\mathrm{n}}}$, was assumed to be 3.00 [24]. Therefore, the $\overline{M_{\mathrm{n}}}$ value of degraded chitosan chain at different reaction times can be calculated from Eq. (13). The results were listed in Table 1. The presumed value of PDI for the chitosan was examined by the re-calculation of this value from the degraded chitosan chains at different reaction times. It was found that the average value is $3.69 \pm 0.98$, indicating that there was no significant difference between the calculated average value and the assumed PDI. This implied that the assumed reaction mechanism is reasonable. In addition, if the calculated $\overline{M_{\mathrm{n}}}$ from Eq. (13) was taken logarithm and then plotted against the retention time measured from the SE-HPLC chromatograms in Fig. 1, a linear plot was observed as shown in Fig. 6. The results are, therefore, in accordance with the widely known equation, $\log \overline{M_{\mathrm{n}}}=-a\left(t_{\mathrm{R}}\right)+b$, which suggested that the theoretical values agreed with

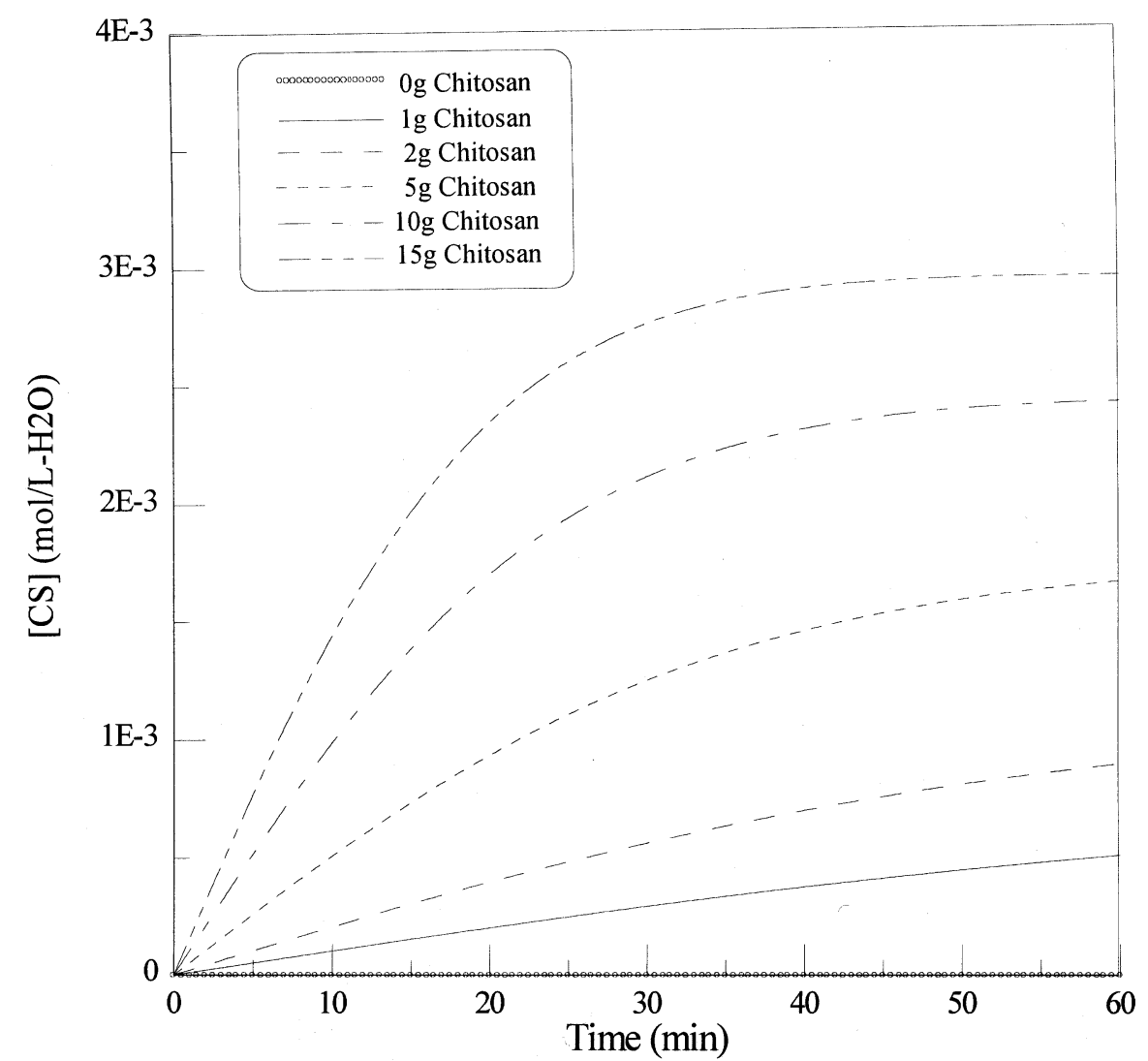

Fig. 5. Theoretical concentrations of degraded chitosan fragment with degradation time at different amounts of chitosan added. KPS was $1.08 \mathrm{~g}$ and the temperature was $70{ }^{\circ} \mathrm{C}$. 


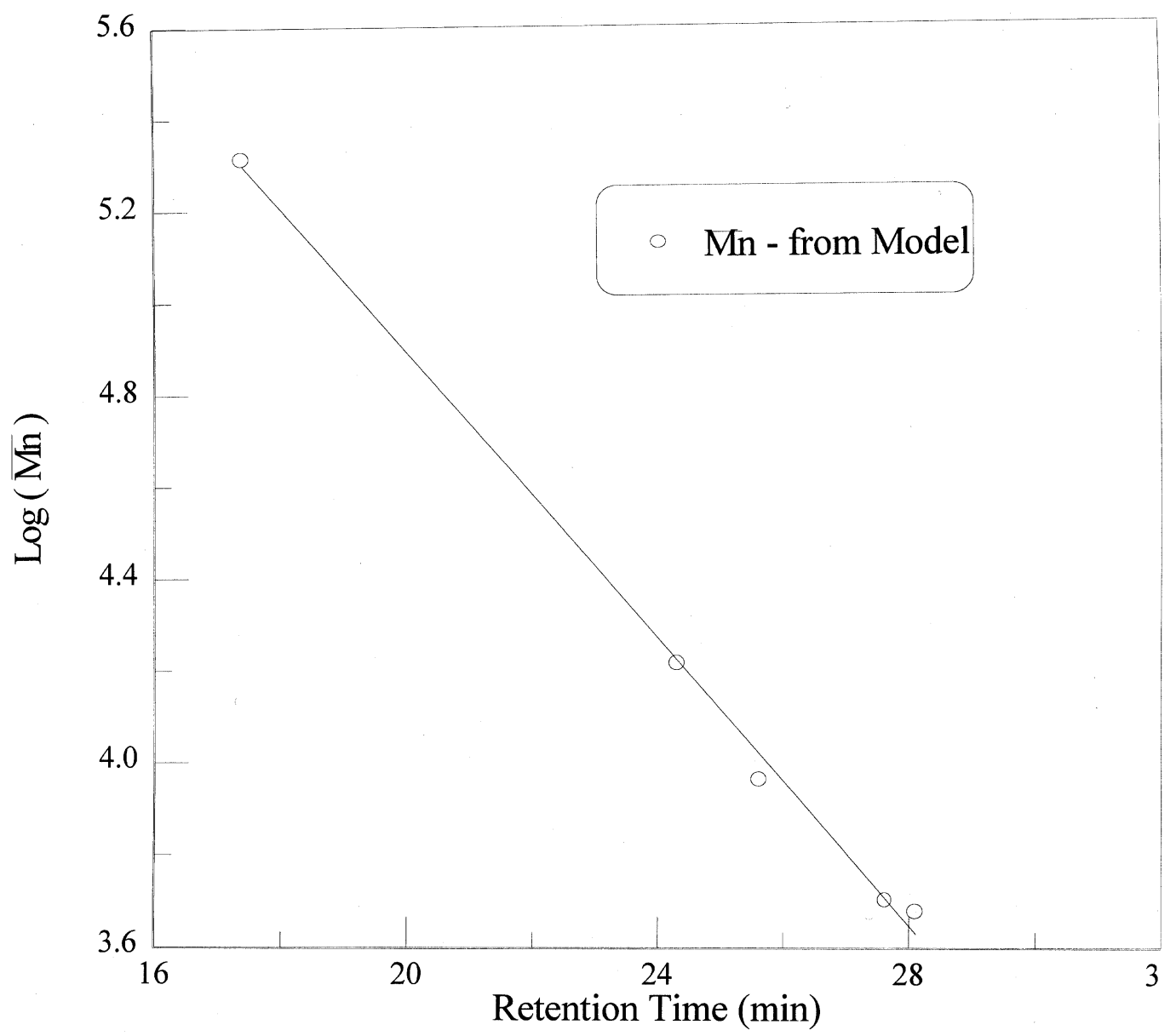

Fig. 6. The logarithm of number average molecular weight versus retention time in GPC chromatogram at different degradation times. $15 \mathrm{~g}$ of chitosan in a $2 \%(\mathrm{w} / \mathrm{w})$ acetic acid solution was degraded by $1.08 \mathrm{~g}$ of KPS at $70{ }^{\circ} \mathrm{C}$.

the experimental ones. In conclusion, the reasonable PDI value in Table 1 and the perfect linear fitting in Fig. 6 strongly suggested that the proposed reaction mechanism and the following calculations are quite reasonable.

\section{Conclusions}

A free radical degradation mechanism of chitosan by KPS was proposed to explain the degradation behavior. Briefly, persulfate ions were thermally dissociated into anionic radicals and attracted to the cationic amino groups at the C-2 carbon of the chitosan ring. Subsequently, the anionic radical attacked the C-4 carbon, and transferred the radical to the C-4 carbon by subtracting the hydrogen from it. The presence of free radical at C-4 carbon finally led to the chain scission of $\mathrm{C}-\mathrm{O}-\mathrm{C}$ bond in the main chain. The calculated average molecular weight of degraded chitosan agreed to the experimental value. In addition, the terminal structures of the two degraded chitosan chain were expected to have effects on the free radicals and initiator, namely, inhibition and deactivation. By solving the simultaneous rate equations according to the proposed mechanism, the concentrations of initiator and total free radicals decreased with time, while the degraded chitosan chain increased. It is concluded that if KPS was used to initiate monomer for polymerization in the presence of chitosan, the degradation behavior of chitosan must be considered, which not only it would affect the emulsion polymerization kinetics, but also the final structures of cationic nanoparticles.

\section{Acknowledgements}

The authors express their appreciation for the financial support of the National Science Council, Republic of China (Project No. NSC 89-2313-B-131-001-A24).

\section{References}

[1] Dumitriu S. Polymeric biomaterials. New York: Dekker, 1994.

[2] Odian G. Principles of polymerization. 3rd ed. New York: Wiley, 1991. 
[3] Colvo P, Remuñán-López C, Vila-Jato JL, Alonson MJ. Colloid Polym Sci 1997;275:46.

[4] Meiser D, Pringle J, Mezei M. Int J Pharm 1989;55:105.

[5] Muzzarelli RAA. Chitin. Oxford: Pergamon Press, 1977.

[6] Stevens WF, Rao MS, Chandrkrachang S. Chitin and chitosan. Bangkok, Thailand: Asian Institute of Technology, 1996.

[7] Chen RH, Chen HC. Advances in chitin science. Keelung, Taiwan: National Taiwan Ocean University, 1998.

[8] Chern CS, Lee CK, Ho CC. Colloid Polym Sci 1999;277:507.

[9] Chern CS, Lee CK, Ho CC. Colloid Polym Sci 1999;277:979.

[10] Yang SC, Ge HX, Hu Y, Jiang XQ, Yang CZ. Colloid Polym Sci 2000;278:285.

[11] Inui H, Kosaki H, Uno Y, Tabata K, Hirano S. Agric Biol Chem 1991;55:3107.

[12] Suzuki K, Mikami T, Okawa Y, Tokoro A, Suzuki S, Suzuki M. Carbohydr Res 1986;151:403.

[13] Aiba S. Carbohydr Res 1994;261:297.
[14] Izume M, Ohtakara A. Agric Biol Chem 1987;51:1187.

[15] Allan GG, Peyron M. Carbohydr Res 1995;277:257.

[16] Chen RH, Chang JR, Shyur JS. Carbohydr Res 1997;299:287.

[17] Toei K, Kohara T. Anal Chim Acta 1975;83:59.

[18] Wang W, Bo S, Li S, Qin W. Int J Biol Macromol 1991;13:281.

[19] Gamzazade AI, Shaishk VM, Sklyer AM, Stikova EV, Pavlova S-SA, Rogozhin SV. Acta Polym 1985;36:420.

[20] Yomota C, Miyazaki T, Okada S. Colloid Polym Sci 1993; 271:76.

[21] Snuparek J, Krska F. J Appl Polym Sci 1977;21:2253.

[22] Snuparek J, Krska F. J Appl Polym Sci 1981;26:4081.

[23] Basset DR, Hoy KL, In: Basset DR, Hamielec AE, editors. Process description and polymer properties in emulsion polymers and emulsion polymerization, ACS Symposium Series 165 . Washington (DC): American Chemical Society, 1981.

[24] Tsaih ML. PhD dissertation, Department of Marine Food Science, National Taiwan Ocean University, Taiwan, 1997. 